LAWRENCE LIVERMORE NAT IO N A L LABORATORY

\section{Photoelectron and X-ray Absorption Spectroscopy Of $\mathrm{Pu}$}

J.G. Tobin, B. Chung, R.K. Schulze, J.D. Farr, D.K. Shuh

November 17, 2003

Fall I2003 MRS

Boston, MA, United States

December 1, 2003 through December 5, 2003 
This document was prepared as an account of work sponsored by an agency of the United States Government. Neither the United States Government nor the University of California nor any of their employees, makes any warranty, express or implied, or assumes any legal liability or responsibility for the accuracy, completeness, or usefulness of any information, apparatus, product, or process disclosed, or represents that its use would not infringe privately owned rights. Reference herein to any specific commercial product, process, or service by trade name, trademark, manufacturer, or otherwise, does not necessarily constitute or imply its endorsement, recommendation, or favoring by the United States Government or the University of California. The views and opinions of authors expressed herein do not necessarily state or reflect those of the United States Government or the University of California, and shall not be used for advertising or product endorsement purposes. 


\title{
Photoelectron and X-ray Absorption Spectroscopy of Pu
}

\author{
J.G. Tobin ${ }^{1, *}$, B.Chung ${ }^{1}$, R. K. Schulze ${ }^{2}$, J. D. Farr ${ }^{2}$, and D. K. Shuh ${ }^{3}$ \\ 1.Lawrence Livermore National Laboratory, Livermore CA, USA \\ 2.Los Alamos National Laboratory, Los Alamos NM, USA \\ 3.Lawrence Berkeley National Laboratory, Berkeley, CA, USA
}

\begin{abstract}
We have performed Photoelectron Spectroscopy and X-Ray Absorption Spectroscopy upon highly radioactive samples of Plutonium at the Advanced Light Source in Berkeley, CA, USA. First results from alpha and delta Plutonium are reported as well as plans for future studies of actinide studies.
\end{abstract}

\section{INTRODUCTION}

The central thrust of this project is to determine which terms in the Hamiltonian for the electronic states of $\mathrm{Pu}$ are dominant. We are moving towards that goal.

The nature of $\mathrm{Pu} 5 \mathrm{f}$ electronic structure is still under debate.[1,2] Many of the complications are derived from the necessity of explaining the phase-specific behavior of $\mathrm{Pu}$ and $\mathrm{Pu}$ alloys, particularly the low symmetry (monoclinic) $\square$ phase and the high symmetry (fcc) $\square$ phase. Experimentally, there are severe hindrances, such as the general lack of single crystals and the radioactive and chemical hazards of the materials. Theoretically, no single model has gained universal acceptance, because of the limitations of each approach. Many of the same questions remain from earlier formulations. Several of these key questions revolve around the interaction of the spin and orbital angular momenta.

Photoelectron Spectroscopy (PES) and X-Ray Absorption Spectroscopy (XAS) have used to investigate the electronic structure of alpha and delta Pu. Additionally, it now appears that a new experiment, based upon "Double Polarization," may be the key to differentiating between the several models now being proposed to explain the electronic structures of alpha and delta $\mathrm{Pu}$. Here the combination of a chiral xray environment and true spin detection will allow us to test whether spin-orbit, exchange, coulombic repulsion or other multielectronic effects drive the differences between alpha and delta $\mathrm{Pu}$.

\section{EXPERIMENTAL}

The first experiments were performed at the Spectromicroscopy Facility (Beamline 7.0) at the Advanced Light Source in Berkeley, CA[3]. The new Pu samples were taken from a specially purified batch of $\mathrm{Pu}$ metal. The plutonium was zone refined and vacuum distilled while magnetically levitated[4]. The product of the purification process was $\square-\mathrm{Pu}$ containing a total of $170 \mathrm{ppm}$ impurities. A portion of the refined metal was alloyed with gallium to form the $\square$-phase (fcc symmetry). The aged sample was less highly purified. The sample surfaces were prepared by repeated room-temperature, sputter-annealing cycles to minimize the amount of oxygen and other impurities 
dissolved in the sample or at grain boundaries, in a specially designed chamber attached to the sample introduction and analysis systems on Beamline 7.0.

\section{DISCUSSION}

Using the tunability of synchrotron radiation, it is possible to perform many variants of photoelectron spectroscopy and x-ray absorption, including accessing the core levels of the sample constituents. For example, sample quality could be confirmed by examining the valence band region at higher photon energies[1]. An example of this is shown in Figure 1.

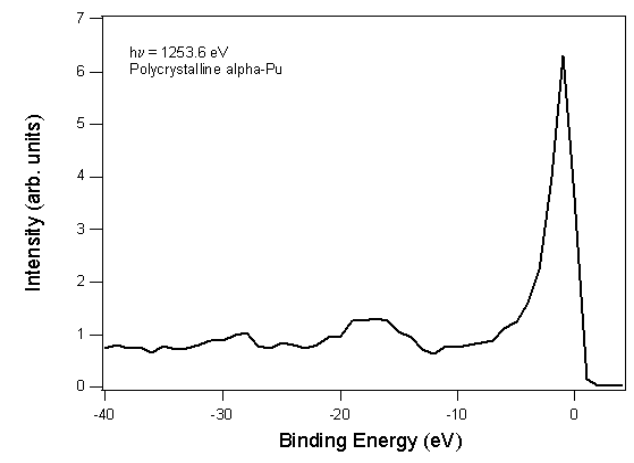

Figure 1. PES valence band spectrum of $\square-\mathrm{Pu}$ at a photon energy of $1250 \mathrm{eV}$.

Another of the variants that was pursued was Resonant Photoemission. Photoelectron spectroscopy is a "photon-in, electron-out" process. Often, it can be simplified down to a single electron phenomenon, where the energy of the photon is absorbed and transferred over entirely to a single electron, while all other "spectator" electrons essentially remain frozen. An advantage of this is its simplicity of interpretation. But in many systems, it is possible to induce a process with heightened sensitivity and significantly increased cross sections: resonant photoemission (ResPes, Ref 1). Here, a second set of indirect channels open up, which contribute in concert with the original or direct channel of simple photoemission. Shown in Figure 2 is a the resonant photoemission results for three $\mathrm{Pu}$ samples, involving the $5 \mathrm{f}$ and $5 \mathrm{~d}$ electrons.

The Pu5f5f5d resonant photoemission process occurs over the photon energy range of $90 \mathrm{eV}$ to $150 \mathrm{eV}$, with an anti-resonance near $100 \mathrm{eV}$ and a resonance maximum near $120 \mathrm{eV}$. In our experiments, both alpha and delta Pu samples have been investigated. The ResPes Spectra exhibit only small differences between the new alpha and the new delta phase samples. These observations correlate well with the results of Gouder et al[5], Arko et al[6] and L.E. Cox[7], which suggest that surface reconstruction may be an important issue in Pu samples. Nevertheless, significant differences can be observed between the new and the aged samples. These results suggest that the valence electronic structure of $\mathrm{Pu}$ is dependent upon its chemical state.

While the RESPES of new and aged samples were significantly different, wide scans containing the core levels and valence bands of each were quite similar, as can be seen in Figure 3 below. 

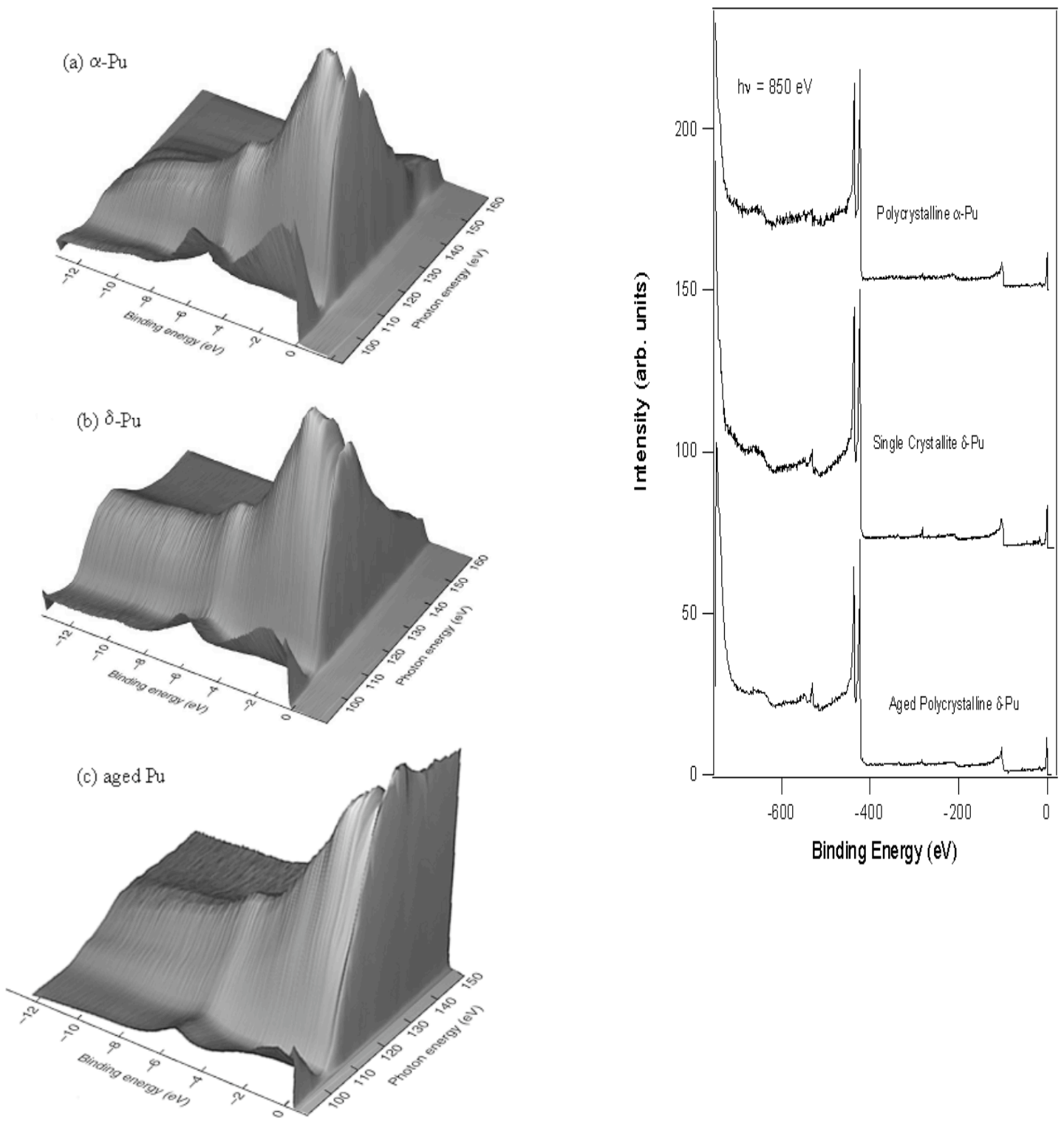

Binding Energy (eV)

Figure 2. Resonant Photoemission or ResPes is a type of spectroscopic interrogation of the valence electronic structure. The results for three samples are shown here: new $\mathrm{\square}-\mathrm{Pu}$, new $\mathrm{P}-\mathrm{Pu}$ and aged $\mathrm{P}-\mathrm{Pu}$. The plots show the intensity variations ( $\mathrm{z}$ axis) versus the binding energy of the states (the negative numbers in eV; zero is the Fermi energy) and photon energy(between $90 \mathrm{eV}$ and $160 \mathrm{eV}$ ).

Figure 3. Comparison of the wide-scan spectra of the three Pu samples at a photon energy of $850 \mathrm{eV}$. 
The similarity of the RESPES results for the new alpha and new delta samples raises an unsettling question. Are the phases actually what we think they are? A means to address this issue and related surface quality questions is to investigate the core level spectroscopy of the $\mathrm{Pu}$. Some our results are shown in Figure 4 below. The spectra in Figure 4 confirm that we have phase specific samples. The 4 f core level PES spectra of the new alpha and new delta samples are each consistent with earlier reported results [811]. Furthermore, it is also possible to access the higher energy $4 \mathrm{~d}$ to $5 \mathrm{f}$ transitions, as shown in Figure 5. The implications of these measurements are discussed in more detail in References 1 and 2.
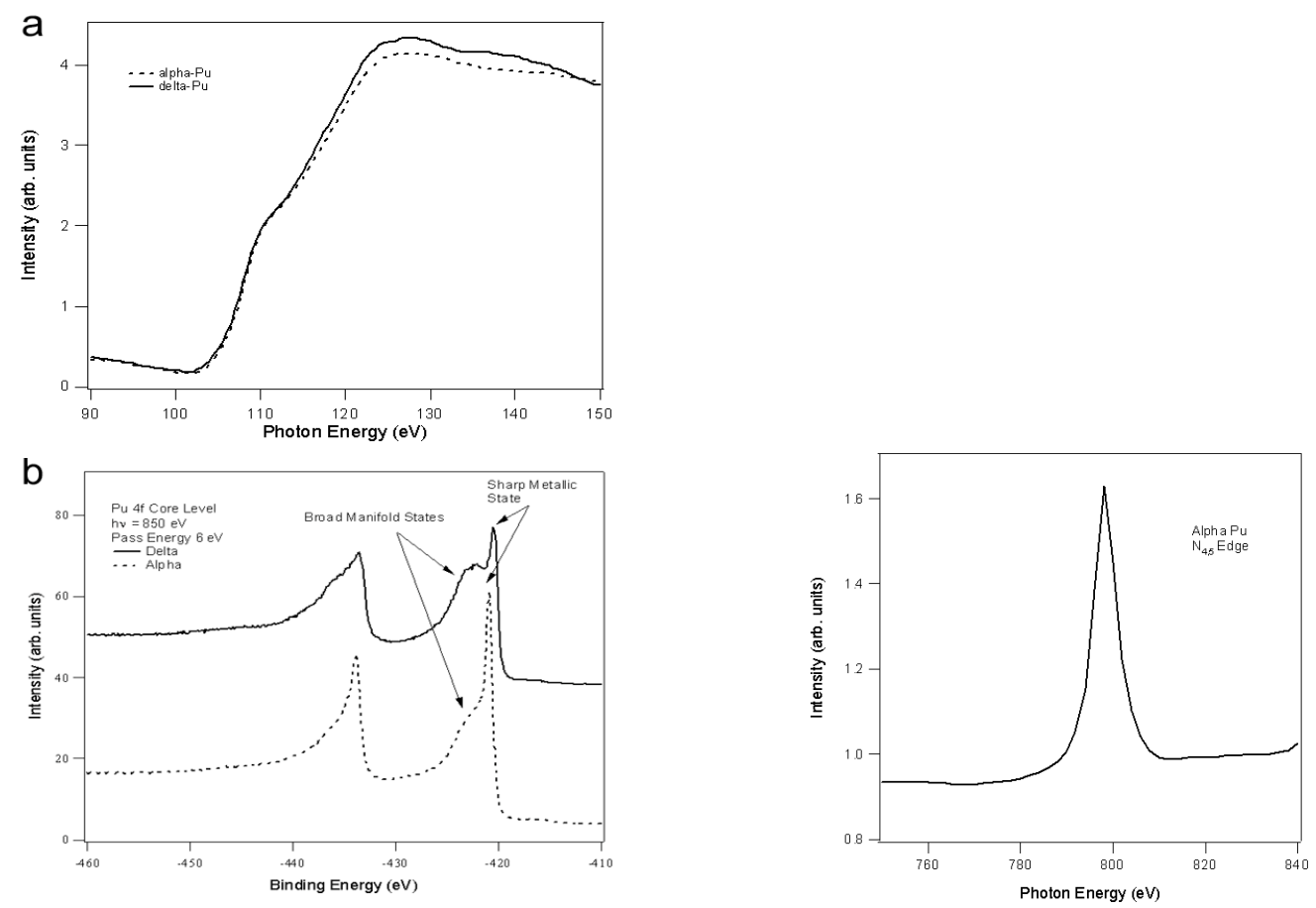

Figure 4. XAS (5d to 5f) and 4f PES spectra that demonstrate phase specificity of the new alpha and new delta samples.

Figure 5. XAS (4d to 5f) of new $\square-\mathrm{Pu}$. Only the $\mathrm{N}_{5}$ edge is shown here.

Another advantage of photon energy tunability is the capability to increase the sensitivity to individual elements by tuning to where their cross section is larger. Below we show that by going from a photon energy of $1250 \mathrm{eV}$ to $860 \mathrm{eV}$, it is possible to enhance our ability to observe the small oxygen concentration of the Pu sample. The wide scan and the detail of the $\mathrm{O} 1 \mathrm{~s}$ region at $1250 \mathrm{eV}$ confirm that our samples are quite "clean." In the future, this tunability will permit us to study surface oxide formation with improved and lower detection limits.

Finally, we are presently constructing a new dedicated Pu Spectrometer, a schematic of which is shown in Figure 6. This spectrometer will include specialized capabilities for handling Pu samples. For example, the long vertical manipulator will allow the isolation of the radioactive byproducts of sample preparation and cleaning 
from the analysis station yet also permit rapid access of the analysis position, so as to minimize surface corruption after cleaning. The photoelectron detection will include capabilities for both multichannel, spin-integrated analysis as well as true spin resolved spectroscopy using a MiniMott detection scheme[12].

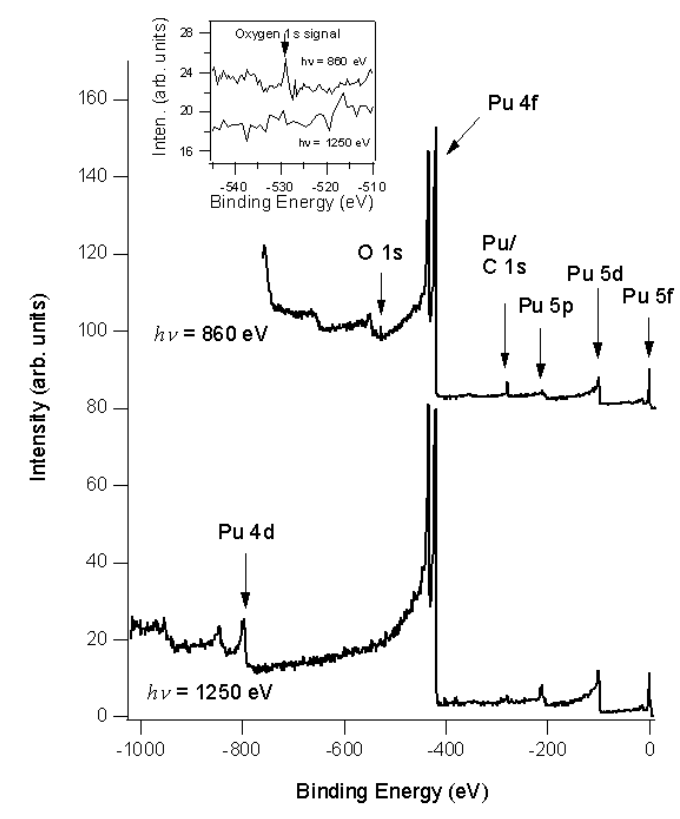

Figure 5 (above) Core level photoelectron spectroscopy: Wide scans at both $1250 \mathrm{eV}$ and $860 \mathrm{eV}$, with a detail of the $\mathrm{O} 1 \mathrm{~s}$ peak region.

Figure 6 (Below)Schematic of the new, dedicated Pu Spectrometer.

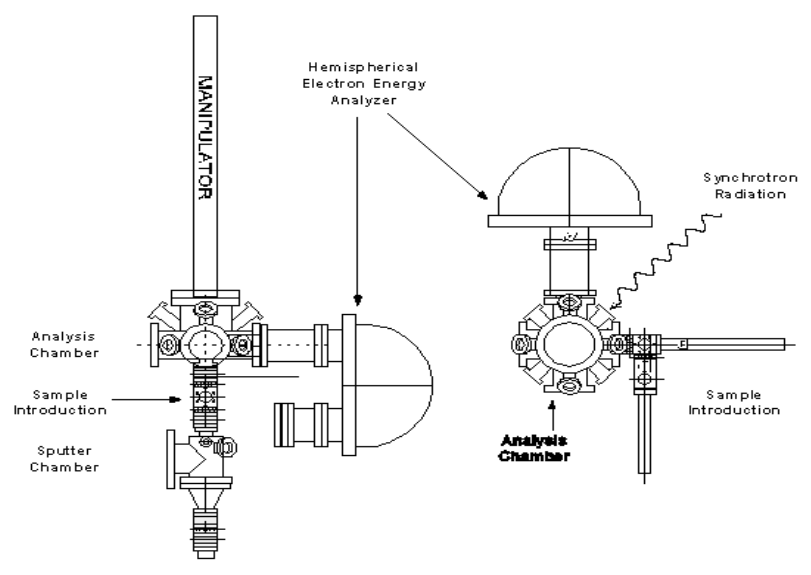




\section{ACKNOWLEDGMENTS}

This work was performed under the auspices of the U.S. Department of Energy by University of California, Lawrence Livermore National Laboratory under Contract W7405-Eng-48.

This work was performed under the auspices of the U.S Department of Energy by LLNL (Contract No. W-7405-Eng-48), LANL (Contract No. W-7405- ENG-36) and LBNL (Contract No. DE-AC03-76SF00098). The Spectromicroscopy Facility (Beamline 7.0) and the Advanced Light Source were built and and are supported by the DOE Office of Basic Energy Research. The authors wish to thank Jason Lashley and Michael Blau for synthesis of the Pu samples.

*Corresponding Author

LLNL, L-357, POB 808, 7000 East Ave.

Livermore, CA, USA; Tobin1@,LLNL.Gov

\section{REFERENCES}

1. J.G. Tobin, B.W. Chung, R. K. Schulze, J. Terry, J. D. Farr, D. K. Shuh, K. Heinzelman, E. Rotenberg, G.D. Waddill, and G. Van der Laan, Resonant Photoemission in f-electron Systems: Pu and Gd", Phys. Rev. B 68, 155109 (2003) and references therein.

2. K.T. Moore, M.A. Wall, A.J. Schwartz, B.W. Chung, D.K. Shuh, R.K. Schulze, and J.G. Tobin, "The Failure of Russell-Saunders Coupling in the $5 \mathrm{f}$ States of Plutonium", Phys. Rev. Lett. 90, 196404 (2003) and references therein.

3. J.D. Denlinger et al, Rev. Sci. Instrum. 66, 1342 (1995).

4. J.C. Lashley, J. Nucl. Matl. 274, 315 (1999).

5. T. Gouder, L. Havela, F. Wastin, and J. Rebizant, Europhys. Lett. 55, 705 (2001).

6. A. Arko et al, Phys. Rev. B 62, 1773 (2000).

7. L.E. Cox et al, Phys. Rev. B 46, 13571 (1992).

8. T. Gouder et al, J. Alloys Cmpds. 271-273, 841 (1998); J. El. Spect. Rel. Phen. 101-105, 419 (2000).

9. R. Baptist et al., J. Phys. F (Metal Phys.) 12, 2103 (1982).

10. L. E. Cox, Phys. Rev. B 37, 8480 (1988).

11. J. R. Naegele, J. Nucl. Matl. 166, 59 (1989).

12. J.G. Tobin and F.O. Schumann, Surf. Sci. 478. 211 (2001); J.G. Tobin et al, MRS Symp. Proc. 524, 185 (1998). 